\title{
Excess protein intake relative to fiber and cardiovascular events in elderly men with chronic kidney disease
}

Hong $\mathrm{Xu}, \mathrm{MD},{ }^{\mathrm{a}}$ Megan Rossi, PhD, BSc, ${ }^{\mathrm{b}, \mathrm{c}}$ Katrina L Campbell, PhD, ${ }^{\text {bd }}$ Gloria Lissete Sencion, MD, ${ }^{\mathrm{a}}$ Johan Ärnlöv, MD, PhD, ${ }^{\mathrm{e}, \mathrm{f}}$ Tommy Cederholm, MD,PhD, ${ }^{\mathrm{g}}$ Per Sjögren, $\mathrm{MD}, \mathrm{PhD},{ }^{\mathrm{g}}$ Ulf Risérus, $\mathrm{PhD},{ }^{\mathrm{g}}$ Bengt Lindholm, MD, $\mathrm{PhD},{ }^{\mathrm{a}}$ Juan Jesús Carrero, $\mathrm{PhD}$,

${ }^{a}$ Divisions of Renal Medicine and Baxter Novum, Department of Clinical Science, Intervention and Technology, Karolinska Institutet, Stockholm, Sweden

${ }^{\mathrm{b}}$ Centre for Kidney Disease Research, Princess Alexandra Hospital, Brisbane, Australia

${ }^{c}$ Division of Diabetes and Nutritional Sciences, King's College, London, United Kingdom

${ }^{\mathrm{d}}$ Bond University, Faculty of Health Sciences and Medicine, Robina, Australia

${ }^{\mathrm{e}}$ Department of Medical Sciences, Molecular epidemiology, Uppsala University, Uppsala, Sweden

${ }^{\mathrm{f}}$ School of Health and Social Studies, Dalarna University, Falun, Sweden

g Department of Public Health and Caring Sciences, Clinical Nutrition and Metabolism, Uppsala University, Uppsala, Sweden

${ }^{\mathrm{h}}$ Center for Molecular Medicine, Karolinska Institutet, Stockholm, Sweden

Running head: Protein-fiber intake ratio, CVD events, CKD

Word counts: Abstract: 250 words, Text: 2806 words, Tables: 2, Figure: 1.

Correspondence to: Juan Jesús Carrero

Divisions of Renal Medicine and Baxter Novum

Karolinska University Hospital, Huddinge M99, Karolinska Institutet

SE-14186 Stockholm, Sweden

Tel.: +46-8-58583982 Fax: +46-8-58583925 


\section{ABSTRACT}

Background and Aims: The elevated cardiovascular (CVD) risk observed in chronic kidney disease (CKD) may be partially alleviated through diet. While protein intake may link to CVD events in this patient population, dietary fiber has shown cardioprotective associations. Nutrients are not consumed in isolation; we hypothesize that CVD events in CKD may be associated with dietary patterns aligned with an excess of dietary protein relative to fiber.

Methods and Results: Prospective cohort study from the Uppsala Longitudinal Study of Adult Men. Included were 390 elderly men aged 70-71 years with CKD and without clinical history of CVD. Protein and fiber intake, as well as its ratio, were calculated from 7-day dietary records. Cardiovascular events were registered prospectively during a median follow-up of 9.1 (inter-quartile range, 4.5-10.7) years. The median dietary intake of protein and fiber was 66.7 (60.7-71.1) and 16.6 (14.519.1) grams/day respectively and the protein-to-fiber intake ratio was 4.0 (3.5-4.7). Protein-to-fiber intake ratio was directly associated with serum C-reactive protein levels. During follow-up, 164 first-time CVD events occurred (incidence rate $54.5 / 1,000$ per year). Protein-fiber intake ratio was an independent risk factor for CVD events [adjusted hazard ratio, HR per standard deviation increase (95\% confidence interval, CI) $1.33(1.08,1.64)]$. Although in opposing directions, dietary protein $[1.18(0.97,1.44)]$, dietary fiber alone $[0.81(0.64,1.02)]$, were not significantly associated with CVD events.

Conclusions: An excess of dietary protein relative to fiber intake was associated with 
the incidence of cardiovascular events in a homogeneous population of older men with CKD.

Key words: protein intake, fiber intake, protein-fiber intake ratio, CVD, CKD 


\section{INTRODUCTION}

Poor lifestyle habits, including unhealthy diet, may contribute to the elevated cardiovascular disease (CVD) risk observed in individuals with chronic kidney disease (CKD) [1]. Excess protein intake has been associated with increased CVD morbidity and mortality in some [2-4] but not all community-based studies [5-7]. For patients with CKD, limiting protein intake remains an important modifiable risk factor for haltering CKD progression, but whether high protein intake is associated with cardiovascular risk in this patient population has not been established. In contrast, observational studies consistently report that a high fiber intake is associated with reduced cardiovascular risk and mortality in the community [8]. Moreover, this association appears to be stronger in adults with $\operatorname{CKD}[9,10]$.

Nutrients are not consumed in isolation. There is a considerable biological ground to support the notion that reducing protein intake and increasing the intake of fruit, vegetables and, in essence, dietary fiber, may mitigate the excess cardiovascular risk of CKD patients [11]. A significant difference in generation rates of two major nephrovascular toxins indoxyl sulfate and p-cresyl sulfate between omnivores and vegetarians has been demonstrated in the healthy population, presumably owing to their different protein and fiber profiles (i.e. higher protein: fiber ratio in omnivores) [12]. Furthermore, we showed that the ratio of dietary protein-fiber intake was strongly associated with the serum concentration of these toxins in CKD patients [13]. However, neither study found an association between the toxins and individual 
nutrients, suggesting the importance of considering the nutrients' combined effect [12, 13]. We hypothesize that dietary patterns aligned with an excess of dietary protein relative to fiber may be more important than the component nutrients on CVD risk in CKD. 


\section{METHODS}

\section{Study population}

This study was performed in the Uppsala Longitudinal Study of Adult Men (ULSAM) (http://www2.pubcare.uu.se/ULSAM/). The present analyses are based on the third ULSAM examination cycle. During this examination, participants were 70-71 years of age (examinations performed during 1991-1995; $n=1221$ ). A total of 692 individuals were identified as having CKD on the basis of cystatin C-estimated glomerular filtration rate $(\mathrm{eGFR})<60 \mathrm{~mL} / \mathrm{min} / 1.73 \mathrm{~m} 2(n=506)$ or urine albumin excretion rate $(\mathrm{UAER}) \geq 20 \mu \mathrm{g} / \mathrm{min}(n=292)$, or both these conditions $(n=106)$. Of those, we excluded individuals with a history of CVD $(n=302)$, defined as any CVD recorded in the Swedish Patient register (Inter-national Classification of Diseases (ICD)-8 codes 390-458 or ICD-9 codes 390-459). The remaining 390 participants were considered for the present study. The Ethics Committee at Uppsala University approved the study and all participants gave informed consent.

\section{Demographics and co-morbidities}

Investigations were performed under standardized conditions. Smoking status was defined as current smoking versus non-smoking. Regular physical activity was defined as the reporting of regular or athletic leisure time exercise habits according to four physical activity categories (sedentary, moderate, regular, and athletic). Blood pressure (BP) was measured in duplicate in the right arm with the subject in the supine position at rest. Hypertension was defined as $\mathrm{BP} \geq 140 / 90 \mathrm{mmHg}$ or the use of 
antihypertensive medications. Diabetes mellitus was defined as fasting plasma glucose $\geq 126 \mathrm{mg} / \mathrm{dL}(7.0 \mathrm{mmol} / \mathrm{L}), 2 \mathrm{~h}$ post-load glucose levels $\geq 200 \mathrm{mg} / \mathrm{dL}(11.1$ $\mathrm{mmol} / \mathrm{L}$ ) or diabetes medication (insulin or oral). Hyperlipidemia was defined as serum cholesterol $>250 \mathrm{mg} / \mathrm{dL}(6.5 \mathrm{mmol} / \mathrm{L})$, serum triglycerides $>200 \mathrm{mg} / \mathrm{dL}(2.3$ $\mathrm{mmol} / \mathrm{L}$ ) or treatment with lipid-lowering medications.

\section{Protein-fiber intake ratio assessment}

Dietary habits were evaluated by a 7-day dietary record based on a validated precoded menu book, which was prepared and previously used by the Swedish National Food Administration (NFA). A dietitian gave the participants verbal instructions of how to complete the dietary record, and the amounts consumed were reported in household measurements or specified as portion sizes. The daily intake of protein and fiber as well as its ratio were calculated using a food composition database from the NFA. Other nutrients that are typically aligned with this ratio and considered in these analyses were saturated fatty acid intake (SFA), potassium and salt intake. Dietary salt was estimated as the natural salt-content of foods and, when reported by the participants, the salt added to cooking and at the table. In order to reduce extraneous variation, nutrient intake was corrected for total energy intake by regression analysis of the residual method [14].

\section{Laboratory measurements}

Venous blood samples were drawn in the morning after an overnight fast and stored at 
$-70{ }^{\circ} \mathrm{C}$ until required for analyses. Serum cystatin C (N Latex Cystatin C, Dade

Behring) was used to estimate GFR by the formula $y=77.24 \times \mathrm{x}^{-1.2623}$, which has been shown to be closely correlated with iohexol clearance in our previous study [15]. Urine albumin excretion rate (UAER) was calculated based on the amount of albumin in the urine collected during the night. The subjects were instructed to void immediately before going to bed and to record the time. All samples during the night and the first sample of urine after rising were collected and used for the analysis with radioimmunoassay kit (Albumin RIA 100; Pharmacia, Uppsala, Sweden). Serum high sensitivity C-reactive protein (hs-CRP) was measured by latex enhanced reagent (Dade Behring, Deerfield, IL) using a Behring BN ProSpec analyzer (Dade Behring).

\section{Follow-up and CVD events incidence}

The analysis of CVD event incidence considered the combined endpoint of both fatal and non-fatal events. The Swedish National vital status Registry and Swedish national hospital discharge register were used to identify CVD events (identified by ICD-9 codes 390-459 or ICD-10 codes I00-I99), and were thereafter validated by inspection of medical records. Patient follow-up was done prospectively from examination date until first CVD events, death by causes other than CVD or December 31, 2003, whichever came first. There was no loss to follow-up.

\section{Statistics}

Values are expressed as mean \pm standard deviation (SD) for normally distributed 
continuous variables, median (inter-quartile range, IQR) for skewed variables, or percentage of total for categorical variables. Spearman's rank correlation analysis was used to determine relationships between protein-fiber intake ratio and variables of interest. The association of protein-fiber intake ratio, and its separate components with CVD event incidence were investigated with Cox proportional-hazard regressions in two sets of models: (i) crude model; (ii) adjusted for age, lifestyle parameters (BMI, smoking status, and physical activity), co-morbidities (prevalence of diabetes, hypertension and hyperlipidemia), renal biomarkers (eGFR and UAER) and food intake (total energy, sodium, potassium and SFA intake). Restricted cubic spline graphs were used to graphically evaluate systematic relationships between protein-fiber intake ratio and fatal/non-fatal CVD incidence. In this analysis, we included the adjusted model. Data are presented as hazard ratios (HR) and 95\% confidence interval (CI). Proportional hazards assumptions were confirmed using Schoenfeld's test [16]. All statistical analyses were performed using statistical software STATA version 13.0 (Stata Corporation, College Station, TX, USA). All tests were two-tailed and $P<0.05$ was considered significant.

\section{RESULTS}

\section{Baseline Characteristics}

Clinical and biochemical characteristics of included participants are shown in Table 1. The median protein-to-fiber intake ratio was 4.0 (3.5-4.7); dietary protein and fiber intakes were, respectively, 66.7 (60.7-71.1) and 16.6 (14.5-19.1) grams/day. Among 
the 390 individuals, 69 (6.7\%) had CKD stage 1-2, 314 (80.5\%) CKD stage 3, and 7

(2\%) had CKD stage 4. Protein-fiber intake ratio correlated with sodium intake (Spearman's rho=0.13, $\mathrm{P}<0.05)$, potassium $(\mathrm{rho}=-0.25, \mathrm{P}<0.001)$, SFA intake (rho=0.40, $\mathrm{P}<0.001)$ and circulating levels of $\mathrm{CRP}(\mathrm{rho}=0.13, \mathrm{P}<0.05)$.

\section{Cox regression models}

During a median follow-up of 9.1 (IQR 4.5-10.7) years, 164 CVD events occurred (incidence rate 54.5/1,000 person-years at risk). These included 43 fatal and 121 nonfatal events. Table 2 presents analyses of the association between protein-to-fiber intake ratio and its components (as continuous variables) and incidence of CVD events. In separate Cox models, and while apparently acting in opposing directions, neither dietary protein nor dietary fiber, associated with this outcome. As a next step we included both exposures in the same Cox model; while opposing statisticallysignificant associations were observed for both nutrient intakes in crude analysis, multivariate adjustment abrogated such significance albeit without much impact on the magnitude of the risk observed. Finally, we modelled the protein-to-fiber intake ratio, which in crude and fully adjusted models, remained an independent predictor of the incidence of CVD events [HR per SD increase (95\% CI) $1.33(1.08,1.64)]$. A spline curve analysis based on HRs of the fully adjusted model confirmed this and positive linear association (Figure 1). 


\section{DISCUSSION}

In this cohort study of elderly men with mild to moderate CKD, and no history of CVD, a higher protein intake relative to fiber intake was associated with increased CVD risk during follow up. Neither dietary protein nor dietary fiber alone associated consistently with these outcomes.

Studies of the link between dietary protein intake and cardiovascular risk in the community have shown somewhat conflicting associations; for example, a large survey of US women failed to associate protein intake (dietary recalls) with the risk of coronary heart disease [5], whereas a Dutch study of men and women free from CKD found an association between dietary protein (estimated as the normalized protein equivalent of nitrogen appearance, nPNA) and cardiovascular events [3]. It may be argued that excess protein intake poses an even greater CVD risk in individuals with CKD due to the inherent metabolic derangements and compromised filtration capacity [17]. However, to the best of our knowledge, no study to date has evaluated the possible link between high protein intake and risk of CVD events in patients with manifest $\mathrm{CKD}$, despite dietary protein intake restriction being a cornerstone management strategy to delay CKD progression [18]. In our study, there was a suggestive link between protein intake and the risk of CVD events, although this did not reach statistical significance.

Fiber intake, on the other hand, has a more established association with reduced risk 
of CVD events and/or mortality in CKD patients [8, 19]. It was notable that fiber intake in our study was relatively low (16.6 g/day) as compared with dietary recommendations such as those from US advocating almost twice that amount (25-38 g/day) [20]. Recently, the strong inverse association observed between fiber intake and mortality risk in the Prevención con Dieta Mediterránea (PREDIMED) trial, which included community-dwelling individuals with high cardiovascular risk, was mainly explained by a protective effect against CVD-specific deaths [21]. The cardioprotective effect of a high dietary fiber intake is likely attributed to health benefits of fiber on intermediate risk factors for CVD such as lowering of blood pressure, waist circumference, body weight, fasting blood glucose and HDLcholesterol [22]. In patients with CKD, however, the putative health-promoting role of a high dietary fiber intake is not well established, and accordingly recommendations regarding fiber intake in renal nutrition guidelines are in general weak or absent. Recently, a higher fiber intake has been linked with a lower death rate in CKD individuals $[9,10]$, but not with CVD-death [10]. Similarly, the current study failed to show an independent association between fiber intake alone and CVD events.

The effects of a single nutrient on health and disease may be influenced, i.e., enhanced or counteracted, by other nutrients. In this study, we hypothesized that the ratio between protein and fiber intake may better discern CVD risk. The rationale for this hypothesis is two-fold: First, these nutrients have been suggested to exert opposite effects on cardiometabolic risk factors $[2-4,8]$ and their intake can therefore 
theoretically be assumed to counteract each other's effects. Second, as the gut microbiota is implicated in the digestion of these nutrients, the balance between the two nutrients may influence the generation of uremic toxicity [23]. More specifically, dietary protein promotes bacterial proteolytic fermentation, with subsequent increased generation of indoles and phenols [24]. In contrast, dietary fiber instead shifts the colonic microbial activity toward saccharolytic fermentation, resulting in decreased generation of protein-derived uremic toxins [24]. We recently established proof of concept for this hypothesis by showing that the ratio between protein and fiber intake was associated with a lower generation of indoxyl sulfate and p-cresyl sulfate in a small cohort of non-dialyzed CKD patients [13]. Mounting experimental and clinical evidence indicates that these uremic toxins are likely to contribute to cardiovascular and bone disease and accelerate the progression of renal disease in CKD patients [25]. The results of the current study are in line with these observations: Interestingly, consideration of both nutrient intakes in the same Cox model yielded opposing directions in their association with the outcome, but with similar risk magnitude (approximately 15\% higher and lower CVD risk for adjusted protein and fiber intake, respectively). The rationale behind this approach was that the model would control confounding by fiber intake on the effect of protein intake on CVD outcomes and vice-versa. We interpret this result as both nutrients similarly associating with the study outcome via other independent mechanisms beyond saccharolytic fermentation, as discussed above. Further, the interrelation between these nutrients in food sources and body homeostasis justifies that they are studied in proportion to each other (e.g. a 
high protein intake will often follow a high fiber intake and vice versa). When modelling their relative dietary intake proportions, we observed that a high protein relative to fiber intake was more strongly and independently associated with the incidence of CVD events. Our observational analysis cannot imply causality, but may serve as a hypothesis generating exercise to stimulate further studies addressing the potential benefits of limiting protein intake while enhancing fiber intake in this patient population. One important limitation in our analysis is our inability to differentiate protein sources in our database; vegetable protein sources are also likely sources of fiber as well as potassium and other nutrients that have anti-atherogenic benefits, and a recent US analysis indeed indicates that both plant protein intake as well as plant protein to total protein ratio associated with reduced mortality in a large population of CKD individuals [26]. The complexity of diet surpasses our simple design, and dietary patterns aligned with an excess of dietary protein relative to fiber, markedly differ in many other macro or micro-nutrients. Foods rich in dietary fiber contain other nutrients with antioxidant and anti-inflammatory properties, and recent trials in CKD patients elegantly demonstrate how base-inducing dietary approaches with fruit and vegetables, may counter acidosis, attenuate inflammation and preserve renal function [27], which may also link to the reduced CVD risk here observed. Likewise, foods rich in animal protein sources are likely higher in other unhealthy nutrients, such as SFA and salt, and contribute little or no fiber, thereby potentially promoting CVD risk. Indeed in our analysis both SFA and salt were positively associated to a higher protein: fiber ratio. Finally, recent epidemiological studies addressing dietary 
quality in CKD patients are also in line with our observations. For instance, diets rich in fruits and vegetables and lower in meat associated with reduced mortality risk in the CKD individuals from Reasons for Geographic and Racial Differences in Stroke (REGARDS) study [28]. Furthermore, adherence to a Healthy eating index [29] or to a Mediterranean dietary pattern [30], patterns both of which favor fiber-rich foods as opposed to meat, have been linked to improved survival and reduced incidence of renal and cardiometabolic complications in individuals with CKD. Finally, Moe et al. [31] demonstrated in a crossover trial that 1-week adherence to a vegetarian diet resulted in a better phosphorus homeostasis control as compared with a meat-rich diet in patients with CKD stage 4. Although the authors rightly allude to the difference in protein-sources to explain the patient's metabolic improvements, the contribution of fiber from the vegetarian diet, may have also played a role.

Strengths of this study include its prospective nature with complete long time followup of a homogenous, community-based cohort, the use of 7-day dietary records, and detailed phenotypic characterization. The homogeneity of participants in this survey (same age, sex, ethnicity and geographical distribution) increases the likelihood of finding unbiased associations, although on the other hand, it renders a selective population that is not necessarily representative of other ages, other ethnicities, or of women. Another factor that may limit the generalizability of the results of the current study is that we excluded individuals with a history of CVD. The reason for this is that they could have modified their diet and lifestyle as a secondary preventive 
strategy and consequently their inclusion would have introduced bias in our analysis.

We acknowledge the lack of information on urinary sodium excretion or serum potassium as additional limitations. We also acknowledge that dietary salt intake is likely underestimated given the subjectivity of self-reporting of salt added to cooking or at the table in our 7-day food records. Further, in dietary recall research it is assumed that diet remains more or less constant over time. However, we cannot exclude the possibility that patients may have changed their diets during follow-up. Finally, although we adjusted for many important confounders in the association of interest, residual confounding cannot be ruled out.

\section{Conclusions}

High protein-fiber intake ratio was associated with higher CVD event incidence risk in a homogeneous population of older men with moderate CKD and free from CVD history. 


\section{ACKNOWLEDGEMENTS}

This work was supported by the grants from the Westman's Foundation. HX is partially supported by Karolinska Institutet faculty for funding of postgraduates (KID). Baxter Novum is the result of a grant from Baxter Healthcare Corporation to Karolinska Institutet.

\section{DISCLOSURES}

BL is affiliated with Baxter Healthcare Corporation. None of the other authors declare any conflict of interest. 


\section{REFERENCES}

1. Jha V, Garcia-Garcia G, Iseki K, Li Z, Naicker S, Plattner B, Saran R, Wang AY, Yang CW: Chronic kidney disease: global dimension and perspectives. Lancet 2013, 382:260-272.

2. Lagiou P, Sandin S, Weiderpass E, Lagiou A, Mucci L, Trichopoulos D, Adami HO: Low carbohydrate-high protein diet and mortality in a cohort of Swedish women. $J$ Intern Med 2007, 261:366-374.

3. Halbesma N, Bakker SJ, Jansen DF, Stolk RP, De Zeeuw D, De Jong PE, Gansevoort RT, Group PS: High protein intake associates with cardiovascular events but not with loss of renal function. J Am Soc Nephrol 2009, 20:1797-1804.

4. Lagiou P, Sandin S, Lof M, Trichopoulos D, Adami HO, Weiderpass E: Low carbohydratehigh protein diet and incidence of cardiovascular diseases in Swedish women: prospective cohort study. BMJ 2012, 344:e4026.

5. Halton TL, Willett WC, Liu S, Manson JE, Albert CM, Rexrode K, Hu FB: Lowcarbohydrate-diet score and the risk of coronary heart disease in women. $N$ Engl J Med 2006, 355:1991-2002.

6. Hu FB, Stampfer MJ, Manson JE, Rimm E, Colditz GA, Speizer FE, Hennekens CH, Willett WC: Dietary protein and risk of ischemic heart disease in women. Am J Clin Nutr 1999, 70:221-227.

7. Buendia JR, Bradlee ML, Singer MR, Moore LL: Diets higher in protein predict lower high blood pressure risk in Framingham Offspring Study adults. Am J Hypertens 2015, 28:372379.

8. Threapleton DE, Greenwood DC, Evans CE, Cleghorn CL, Nykjaer C, Woodhead C, Cade JE, Gale CP, Burley VJ: Dietary fibre intake and risk of cardiovascular disease: systematic review and meta-analysis. BMJ 2013, 347:f6879.

9. Krishnamurthy VM, Wei G, Baird BC, Murtaugh M, Chonchol MB, Raphael KL, Greene T, Beddhu S: High dietary fiber intake is associated with decreased inflammation and allcause mortality in patients with chronic kidney disease. Kidney Int 2012, 81:300-306.

10. Xu H, Huang X, Risérus U, Krishnamurthy VM, Cederholm T, Arnlöv J, Lindholm B, Sjögren P, Carrero JJ: Dietary fiber, kidney function, inflammation, and mortality risk. Clin J Am Soc Nephrol 2014, 9:2104-2110.

11. Packard DP, Milton JE, Shuler LA, Short RA, Tuttle KR: Implications of chronic kidney disease for dietary treatment in cardiovascular disease. J Ren Nutr 2006, 16:259-268.

12. Patel KP, Luo FJ, Plummer NS, Hostetter TH, Meyer TW: The production of p-cresol sulfate and indoxyl sulfate in vegetarians versus omnivores. Clin J Am Soc Nephrol 2012, 7:982-988.

13. Rossi M, Johnson DW, Xu H, Carrero JJ, Pascoe E, French C, Campbell KL: Dietary protein-fiber ratio associates with circulating levels of indoxyl sulfate and p-cresyl sulfate in chronic kidney disease patients. Nutr Metab Cardiovasc Dis 2015.

14. Willett WC, Howe GR, Kushi LH: Adjustment for total energy intake in epidemiologic studies. Am J Clin Nutr 1997, 65:1220S-1228S; discussion 1229S-1231S.

15. Larsson A, Malm J, Grubb A, Hansson LO: Calculation of glomerular filtration rate expressed in $\mathbf{m L} / \mathbf{m i n}$ from plasma cystatin $\mathbf{C}$ values in $\mathbf{m g} / \mathbf{L}$. Scand $\mathbf{J}$ Clin Lab Invest 
2004, 64:25-30.

16. Ananth CV, Kleinbaum DG: Regression models for ordinal responses: a review of methods and applications. Int J Epidemiol 1997, 26:1323-1333.

17. Friedman AN: High-protein diets: potential effects on the kidney in renal health and disease. Am J Kidney Dis 2004, 44:950-962.

18. Fouque D, Laville M: Low protein diets for chronic kidney disease in non diabetic adults. Cochrane Database Syst Rev 2009:CD001892.

19. Wang X, Ouyang Y, Liu J, Zhu M, Zhao G, Bao W, Hu FB: Fruit and vegetable consumption and mortality from all causes, cardiovascular disease, and cancer: systematic review and dose-response meta-analysis of prospective cohort studies. $B M J$ 2014, 349:g4490.

20. Dietary guidelines for Americans. U.S. Government Printing Office, Washington (DC) (2010); 2010.

21. Buil-Cosiales P, Zazpe I, Toledo E, Corella D, Salas-Salvadó J, Diez-Espino J, Ros E, Fernandez-Creuet Navajas J, Santos-Lozano JM, Arós F, et al: Fiber intake and all-cause mortality in the Prevención con Dieta Mediterránea (PREDIMED) study. Am J Clin Nutr 2014, 100:1498-1507.

22. Estruch R, Martínez-González MA, Corella D, Basora-Gallisá J, Ruiz-Gutiérrez V, Covas MI, Fiol M, Gómez-Gracia E, López-Sabater MC, Escoda R, et al: Effects of dietary fibre intake on risk factors for cardiovascular disease in subjects at high risk. J Epidemiol Community Health 2009, 63:582-588.

23. Consortium HMP: Structure, function and diversity of the healthy human microbiome. Nature 2012, 486:207-214.

24. Meijers BK, Evenepoel P: The gut-kidney axis: indoxyl sulfate, p-cresyl sulfate and CKD progression. Nephrol Dial Transplant 2011, 26:759-761.

25. Niwa T: Indoxyl sulfate is a nephro-vascular toxin. J Ren Nutr 2010, 20:S2-6.

26. Chen X, Wei G, Jalili T, Metos J, Giri A, Cho ME, Boucher R, Greene T, Beddhu S: The Associations of Plant Protein Intake With All-Cause Mortality in CKD. Am J Kidney Dis 2015.

27. Goraya N, Simoni J, Jo C, Wesson DE: Dietary acid reduction with fruits and vegetables or bicarbonate attenuates kidney injury in patients with a moderately reduced glomerular filtration rate due to hypertensive nephropathy. Kidney Int 2012, 81:86-93.

28. Gutiérrez OM, Muntner P, Rizk DV, McClellan WM, Warnock DG, Newby PK, Judd SE: Dietary patterns and risk of death and progression to ESRD in individuals with CKD: a cohort study. Am J Kidney Dis 2014, 64:204-213.

29. Dunkler D, Dehghan M, Teo KK, Heinze G, Gao P, Kohl M, Clase CM, Mann JFE, Yusuf S, Oberbauer R, Investigators O: Diet and Kidney Disease in High-Risk Individuals With Type 2 Diabetes Mellitus. Jama Internal Medicine 2013, 173:1682-1692.

30. Huang XY, Jimenez-Moleon JJ, Lindholm B, Cederholm T, Arnlov J, Riserus U, Sjogren P, Carrero JJ: Mediterranean Diet, Kidney Function, and Mortality in Men with CKD. Clinical Journal of the American Society of Nephrology 2013, 8:1548-1555.

31. Moe SM, Zidehsarai MP, Chambers MA, Jackman LA, Radcliffe JS, Trevino LL, Donahue SE, Asplin JR: Vegetarian compared with meat dietary protein source and phosphorus homeostasis in chronic kidney disease. Clin J Am Soc Nephrol 2011, 6:257-264. 


\section{FIGURE LEGEND}

Figure 1. Restricted cubic spline curve showing adjusted hazard ratios (bold line) and 95\% confidence intervals (dashed lines) for cardiovascular disease incidence associated with protein-fiber ratio. Covariates include age, BMI, smoking status, physical activity, hypertension, diabetes, hyperlipidemia, eGFR, UAER, total energy intake, sodium, potassium and saturated fatty acid intake. The histogram is the distribution of protein-fiber among 390 older men with CKD. The median value served as reference (knot). 
Table 1.Baseline characteristics and univariate correlates of protein-fiber ratio in 390 older men with CKD and free from CVD history.

\begin{tabular}{lcc}
\hline Parameters & & Univariate rho \\
\hline $\mathrm{n}$ & 390 & - \\
Protein-fiber ratio & $71.0 \pm 0.6$ & 0.09 \\
Age, years & $26.3 \pm 3.4$ & 0.02 \\
BMI, kg/m ${ }^{2}$ & $92(24 \%)$ & 0.07 \\
Smokers, $(\%)$ & & -0.05 \\
Physical activity, $\mathrm{n}(\%)$ & $13(3 \%)$ & \\
$\quad$ Sedentary & $143(37 \%)$ & \\
$\quad$ Moderate & $201(52 \%)$ & \\
$\quad$ Regular & $20(5 \%)$ & 0.09 \\
$\quad$ Athletic & $58(15 \%)$ & 0.07 \\
Diabetes mellitus, $\mathrm{n}(\%)$ & $270(69 \%)$ & -0.05 \\
Hypertension, $\mathrm{n}(\%)$ & $131(34 \%)$ & -0.04 \\
Hyperlipemia, $n$ (\%) & $54.4(47.9-58.3)$ & -0.01 \\
eGFR, mL/min per $1.73 \mathrm{~m}^{2}$ & $6.6(3.4-22.8)$ & $0.13^{*}$ \\
UAER, ug/min & $1.9(0.9,4.4)$ & \\
CRP, mg/L & & -0.01 \\
Food intake & $1724(1435-2003)$ & $0.13^{*}$ \\
Energy intake (kcal /day) & $2.5(2.2-2.7)$ & $-0.25^{* * *}$ \\
Dietary sodium (g/day) & $2.8(2.5-3.0)$ & $0.40 * * *$ \\
Dietary potassium (g/day) & $29.6(26.2-33.4)$ & - \\
Dietary SFA (g/day) & $16.6(14.5-19.1)$ & - \\
Dietary fiber (g/day) & $66.7(60.7-71.1)$ & \\
Dietary protein (g/day) & & \\
\hline Data & & \\
\hline
\end{tabular}

${ }^{1}$ Data are expressed as mean \pm standard deviation, median (25th-75th percentile), or number (percentage), as appropriate

${ }^{2}$ Abbreviations: BMI, body mass index; eGFR, estimated glomerular filtration rate; UAER, urinary albumin excretion rate; CRP, c-reactive protein; SFA, saturated fatty acid;

${ }^{3}$ Statistically significant association, $* \mathrm{p}<0.05, * * \mathrm{p}<0.01, * * * \mathrm{p}<0.001$ 
Table 2. Hazard ratios for cardiovascular event incidence ( $\mathrm{n}=164$ events) associated to $1 \mathrm{SD}$ higher dietary intake of protein and fiber, and its ratio, among 390 older men with CKD and free from CVD history.

Crude

Adjusted $^{1}$

Exposure

$\begin{array}{llll}\text { HR }(95 \% \mathrm{CI}) & \mathrm{P} & \mathrm{HR}(95 \% \mathrm{CI}) & \mathrm{P}\end{array}$

\section{A) Single Cox models}

Dietary protein alone

(per SD $8.37 \mathrm{~g} /$ day higher)

$1.15(0.98,1.34)$

$0.09 \quad 1.18(0.97,1.44)$

0.10

Dietary fiber alone

(per SD $3.98 \mathrm{~g} /$ day higher)

$0.82(0.69,0.97)$

$0.02 \quad 0.81(0.64,1.02)$

0.07

\section{B) Joint Cox models}

Protein intake

(per SD $8.37 \mathrm{~g} /$ day higher)

Fiber intake

$1.16(1.00,1.36)$

0.05

$1.14(0.93,1.41)$

0.20

(per SD $3.98 \mathrm{~g} /$ day higher)

$\begin{array}{llll}0.81(0.68,0.96) & 0.01 & 0.83(0.65,1.05) & 0.12\end{array}$

Protein-fiber intake ratio

(per SD 1.15 unit higher)

$1.26(1.11,1.44)$

0.001

$1.33(1.08,1.64)$

0.01

${ }^{1}$ Adjusted for age, BMI, smoking status, physical activity, hypertension, diabetes, hyperlipidemia, eGFR, UAER, total energy intake, sodium, potassium and saturated fatty acid intake. 


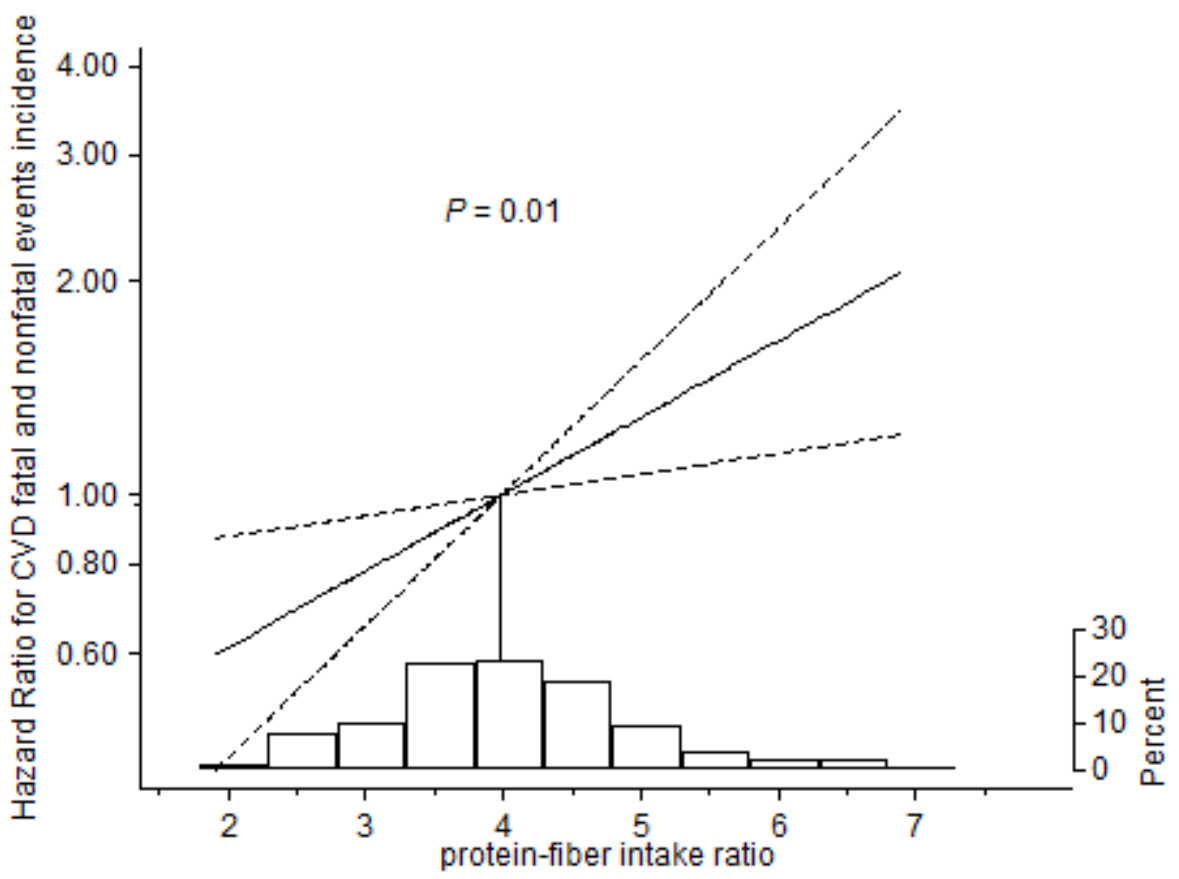

Figure 1. 


\section{HIGHLIGHTS}

- A dietary pattern aligned with an excess of dietary protein relative to fiber strongly associates with the risk of cardiovascular events in a CKD population.

- Neither dietary protein nor dietary fiber alone were consistently associated with this outcome.

- Reducing protein intake and increasing the intake of fruit, vegetables and, in essence, dietary fiber, may mitigate the excess cardiovascular risk of CKD patients. 